\title{
Análise do comportamento de uma rede IEEE 802.15.4 utilizando 6LowPAN
}

\author{
Gabriel V. da S. dos Santos ${ }^{1}$, Pedro A. G. da Fonseca ${ }^{1}$, Samuel F. Ferrigo ${ }^{1}$ \\ ${ }^{1}$ Centro Universitário UNIFTEC \\ Caxias do Sul - RS - Brasil \\ \{gabriel.vinhola, pedro.fonseca, samuelferrigo\}acad.ftec.com.br
}

Resumo. O 6LowPAN é um protocolo que permite que redes IPv6 comuniquemse nativamente com dispositivos de uma rede IEEE 802.15.4. O presente trabalho analisa o consumo energético e a latência de dispositivos IEEE 802.15.4 que utilizam o protocolo 6LowPAN. Como principal resultado obtido, verificouse que o comportamento dos dispositivos analisados que realizavam funções de roteamento apresentaram um maior consumo energético.

\section{Introdução}

As Wireless Personal Area Network (WPAN) podem ser usadas em diversos tipos de aplicações, como no monitoramento da saúde de um indivíduo, rastreamento em ambientes internos, entre outros, assim sendo considerada uma rede de sensores sem fio (RSSF) [Loureiro et al. 2003]. Para poupar energia na comunicação de dados entre os dispositivos, os padrões Bluetooth Low Energy (BLE) e IEEE 802.15.4 utilizam basicamente as mesmas técnicas: longos períodos de inatividade na comunicação - também chamado de baixo ciclo de trabalho - e quadros com payload de poucos bytes [Zanella et al. 2020]. A análise destes sensores demonstram grande importância para a aplicação real de monitoramento de onde quer que seja aplicada esta tecnologia. Assim sendo, este trabalho demonstrará o comportamento destes sensores através de vários testes dentro do simulador, utilizando parâmetros de consumo energético, tamanho, quantidade de mensagens enviadas e latência.

\section{IEEE 802.15.4 e 6LOWPAN}

O padrão IEEE 802.15.4 nos fornece um baixo custo e menor consumo energético na rede. Esse padrão utliza o protocolo CSMA/CA para efetuar o controle de acesso ao meio compartilhado, de forma a evitar colisões [Tolani et al. 2021].

Esses dispositivos - também chamados de motes - podem ser divididos em duas categorias: Full-Function Device (FFD) e Reduced-Function Device (RFD). FFD é um dispositivo completo. Possui todas as funções necessárias às tarefas de roteamento e inicialização da rede. RFD é um dispositivo com recursos limitados. Apresenta apenas funções básicas de ingresso e comunicação na rede [Tolani et al. 2021].

Os FFDs funcionam tanto na topologia estrela como na peer-to-peer (P2P), podendo assim coordenar uma rede e se comunicar com outro dispositivo. Já RFDs estão limitados à topologia estrela e, ao contrário do FFD, não são capazes de coordenar redes e nem se comunicar com outro dispositivo. Estes dispositivos tem menor capacidade de memória e processamento, sendo assim, são mais simples e tem um custo mais baixo, e garantem ainda um menor consumo energético. 
Todavia, o padrão não é nativamente compatível com o padrão TCP/IP. Uma alternativa para contornar essa limitação é o uso do protocolo 6LowPAN, criado pela Internet Engineering Task Force (IETF) que permite a transmissão de pacotes IPv6 em redes que não o suportam nativamente [Geethamani et al. 2021]. Assim como o IEEE 802.15.4, é também focado especialmente para uso em redes sem fio de baixa potência e baixa taxa de transferência de dados [Ferrigo et al. 2017].

O 6LowPAN, inclusive, agrega novas funcionalidades a uma rede IEEE 802.15.4. Uma dessas funcionalidades é o roteamento de quadros dentro dessa rede, de forma que dispositivos possam se comunicar através de um ou mais dispositivos intermediários [Ferrigo et al. 2017]. Assim, através do 6LowPAN, a própria rede IEEE 802.15.4 tornase apta a realizar o roteamento de quadros dentro dela, sem necessidade de um protocolo adicional que responsabilize-se por essa função.

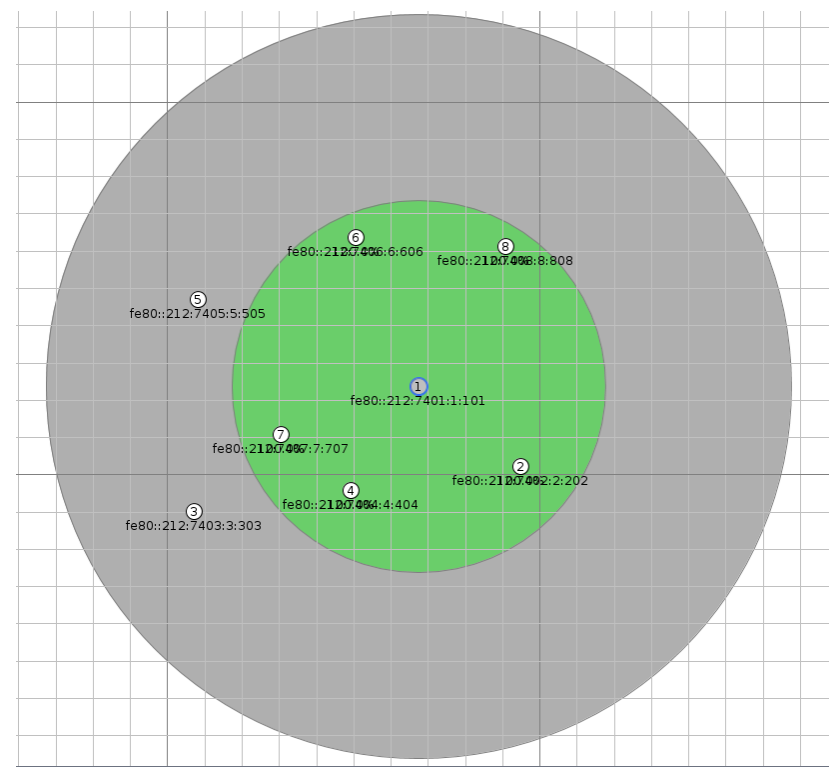

Figura 1. Disposição dos sensores/dispositivos/motes no simulador Cooja.

\section{Cenário}

Para efetuar o estudo, foram utilizados os seguintes dispositivos e equipamentos: uma Virtual Machine (VM) com sistema operacional Ubuntu, contendo a suíte Contiki ${ }^{1}$ com o emulador Cooja ${ }^{2}$, memória de vídeo principal de 16MB, controladora gráfica VboxVGA. A VM foi executada em um computador que contava com um processador Ryzen $73700 x$ de 8 núcleos e 16 threads, com 16 GB de memória RAM, e com uma placa de vídeo RX $5700 \mathrm{XT}$.

Optou-se pelo Cooja pois é uma ferramenta voltada para simulações de uso desta tecnologia e também foi utilizada na disciplina de Novas Tecnologias em Redes de Computadores ofertada pela UNIFTEC. Esse simulador também permite mostrar a utilização de sensores RSSF, contemplando suas interações. A ferramenta executa a rede WPAN mostrada na Figura 1. Essa rede é formada por 8 dispositivos modelo Sky que executam

\footnotetext{
${ }^{1}$ Framework voltado para desenvolivmento e testes de dispostivos de baixo consumo energético

${ }^{2}$ Simulador integrado ao Contiki para execução de RSSF
} 
a função de sensor de medição de temperatura. A comunicação da rede com a Internet é feita pelo sensor 1, que é o dispositivo FFD da rede. Este realiza a função de roteador de borda entre a VM Contiki e os demais dispositivos RFD da rede WPAN. Dessa maneira, através do compartilhamento da rede da VM, qualquer dispositivo ligado à Internet podese comunicar a rede WPAN, conforme mostrado na Figura 2. Todos os dispositivos foram dispostos de forma aleatória, sendo que os sensores 3 e 5 não comunicam-se diretamente com o sensor 1, utilizando-se dos sensores 4, 6 e 7 para realizar tal tarefa. Por fim, todos os dispositivos dessa rede foram configurados para utilizar o 6LowPAN.

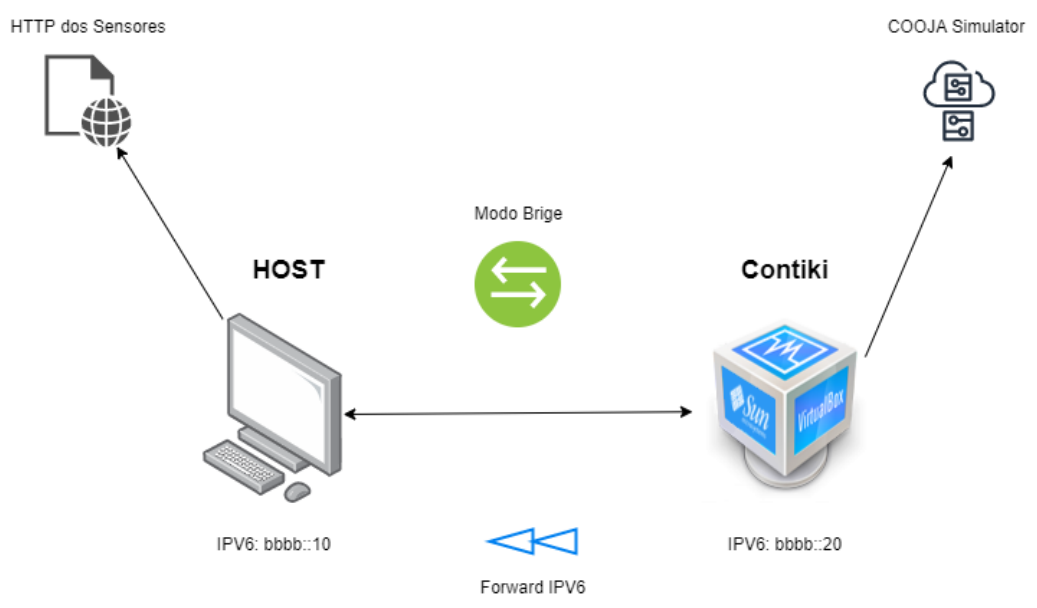

Figura 2. Estrutura do Cenário.

\section{Testes e resultados}

Para realizar os testes, foram realizados pings contendo payload de 32 bytes através do host externo mostrado na Figura 2 para cada um dos motes 1 à 8 identificados na Figura 1 durante 3 minutos. Esse tempo foi assim definido em função de limitações de execução do simulador por períodos superiores a 3 minutos, cuja causa não foi identificada até a confecção deste trabalho. O consumo energético foi estimado através do uso do rádio, utilizando a fórmula aplicada em [Ferrigo et al. 2017].

\begin{tabular}{|l|r|r|r|}
\hline Mote & Consumo Energético (mW) & Radio On (média) & Latência (ms) \\
\hline Sky 1 & 15,6 & 100 & 99 \\
\hline Sky 2 & 6,6 & 0,11 & 102 \\
\hline Sky 3 & 37,2 & 0,77 & 94 \\
\hline Sky 4 & 9,0 & 0,04 & 92 \\
\hline Sky 5 & 9,0 & 0,12 & 326 \\
\hline Sky 6 & 22,8 & 0,02 & 338 \\
\hline Sky 7 & 51,0 & 0,01 & 230 \\
\hline Sky 8 & 30,0 & 0,04 & 243 \\
\hline Average & 22,65 & 0,15 & 191 \\
\hline
\end{tabular}

Tabela 1. Consumo energético, taxa de uso do rádio e latência média de cada um dos dispositivos/motes.

Verificou-se, primeiramente, que o 6LowPAN funcionou satisfatoriamente, pois conseguiu-se alcançar todos os dispositivos WPAN através de um equipamento conectado à Internet. Conforme demontrado na Figura 3, verificou-se um maior consumo energético 
do dispositivo 7, pois este era usado como um intermediário de comunicação entre o dispositivo 3 - e, em alguns casos, do dispositivo 5 - e o dispositivo 1 da rede WPAN. Isso comprova que o funcionamento de roteamento de quadros do 6LowPAN explicado na Seção 2 funciona, porém aumenta o consumo energético do mote que realiza essa função. Nos testes executados, houve um aumento no consumo de cerca de $25 \%$.

Percebeu-se, também, que a maior latência foi verificada no sensor 6, com 338ms, e a menor latência, de $92 \mathrm{~ms}$, foi verificada no mote 4. Isso demonstra que a posição do dispositivo na rede não impacta nesse indicador. $\mathrm{O}$ que de fato interfere na latência é o tráfego da rede em um determinado momento, de acordo com o funcionamento do protocolo CSMA/CA.

\section{Conclusão}

Verificou-se, primeiramente, o correto funcionamento do protocolo 6LowPAN em redes IEEE 802.15.4. Isso permitiu que um dispositivo externo conectado à Internet utilizando IPv6 pudesse ter acesso nativo a essa rede. Além disso, como esperado, os dispositivos com maior consumo energético foram aqueles que tinham a função de roteamento dentro da rede IEEE 802.15.4. Por fim, percebeu-se que o posicionamento dos motes não interferiu na latência.

Como sugestão de trabalhos futuros pode-se realizar testes considerando o uso de outros protocolos de aplicação, de forma a analisar o comportamento da rede utilizando casos de estudo mais próximos de um ambiente real. Também pretende-se verificar o motivo pelo qual a ferramenta Cooja apresentava falhas e travamentos em períodos de execução superiores a 3 minutos.

\section{Referências}

Ferrigo, S. F., da Costa, C. A., and da Rosa Righi, R. (2017). Um middleware para gerenciamento de sensores de saúde corporais em dispositivos móveis. In Anais do IX Simposio Brasileiro de Computacao Ubiqua e Pervasiva. SBC.

Geethamani, R., Karthik, T., Deivakani, M., Jain, V., Mohan, A., Chopra, M., Mahapatra, C., and Manjunath, T. (2021). Implementation of wireless home-based automation and safety arrangement using power electronic switches. Materials Today: Proceedings.

Loureiro, A., Nogueira, J. M. S., Ruiz, L. B., Mini, R. A. D. F., Nakamura, E. F., and Figueiredo, C. M. S. (2003). Redes de Sensores Sem Fio. XXI Simpósio Brasileiro de Redes de Computadores, pages 179-226.

Tolani, M., Sharma, S., and Singh, R. K. (2021). A markov model for ieee 802.15. 4 mac protocol with energy-efficient gts utilization under saturated and unsaturated traffic conditions. Ad Hoc Networks, page 102452.

Zanella, M., Santos, M., Piccoli, R., and Ferrigo, S. (2020). Uso experimental de 6lowpan em redes ble. In Anais da XX Escola Regional de Alto Desempenho da Região Sul, pages 21-24, Porto Alegre, RS, Brasil. SBC. 